\title{
MicroRNA-705 regulates the differentiation of mouse mandible bone marrow mesenchymal stem cells
}

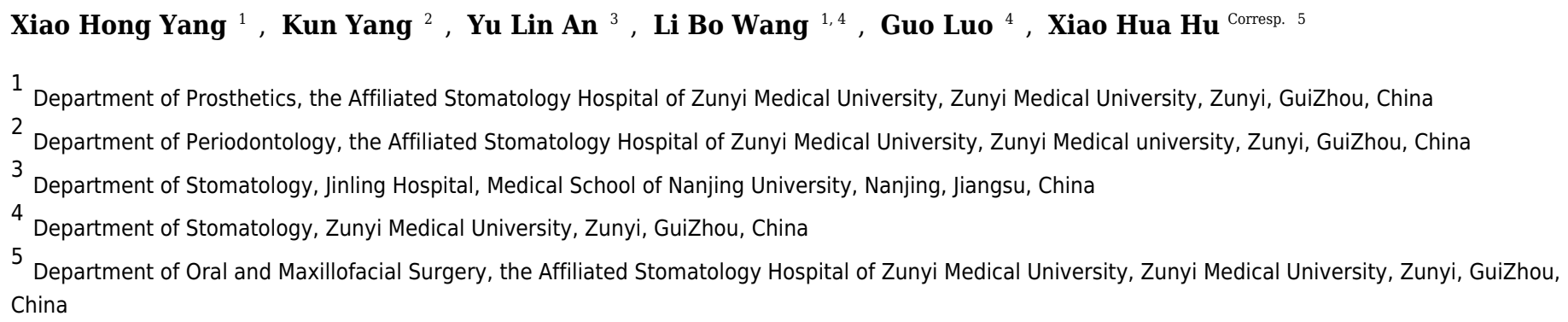

The craniofacial skeleton is the foundation of most stomatological treatments, including prosthodontics and maxillofacial surgery. Although histologically similar to the appendicular skeleton, the craniofacial skeleton manifests many unique properties in response to external stimuli and signals. However, the mandibular or maxillary bone marrow mesenchyme, which is the intrinsic foundation of the functions of craniofacial skeleton, has not been well studied, and its homeostasis mechanism remains elusive. Osteoporosis is a systemic disease that affects all skeletons and is characterized by bone mass loss. Osteoporotic bone marrow mesenchymal stem cells (BMMSCs) exhibit disturbed homeostasis and distorted lineage commitment. Many reports have shown that microRNAs (miRNAs) play important roles in regulating MSCs homeostasis. Here, to obtain a better understanding of mandibular bone marrow MSCs homeostasis, we isolated and cultured mandible marrow MSCs from mouse mandibles. Using miR-705 mimics and an inhibitor, we demonstrated that miR-705 played a vital role in shifting the mandibular MSCs lineage commitment in vitro. Utilizing an osteoporosis mouse model, we demonstrated that MSCS from ovariectomized (OVX) mouse mandibular bone marrow exhibited impaired osteogenic and excessive adipogenic differentiation. miR-705 was found overexpressed in OVX mandibular MSCs. The knock down of miR-705 in vitro partially attenuated the differentiation disorder of the OVX mandibular MSCs by upregulating the expression of osteogenic marker genes but suppressing adipogenic genes. Taken together, our findings provide a better understanding of the homeostasis mechanism of mandibular BMMSCs and a novel potential therapeutic target for treating mandibular osteoporosis. 


\section{MicroRNA-705 regulates the differentiation of mouse} mandibular bone marrow mesenchymal stem cells

Xiao Hong Yang ${ }^{1}$, Kun Yang ${ }^{2 \#}$, Yu Lin An ${ }^{3}$, Li Bo Wang ${ }^{1}$, Guo Luo ${ }^{4}$, Xiao Hua Hu ${ }^{5 *}$

1. Department of prosthetics, the Affiliated Stomatology Hospital of Zunyi Medical University, ZunYi, Guizhou, China

2. Department of Periodontology, the Affiliated Stomatology Hospital of Zunyi Medical University, ZunYi, Guizhou, China

3. Department of Stomatology, Jinling Hospital, Medical School of Nanjing University, Nanjing, Jiangsu, China

4. Department of Stomatology, Zunyi Medical University, ZunYi, GuiZhou, China

5. Department of Oral and Maxillofacial Surgery, the Affiliated Stomatology Hospital of Zunyi Medical University, ZunYi, GuiZhou, China

\# Xiaohong Yang and Kun Yang contributed equally to this work.

*Corresponding author, Email: hxh2132000@163.com

\section{Abstract}

The craniofacial skeleton is the foundation of most stomatological treatments, including prosthodontics and maxillofacial surgery. Although histologically similar to the appendicular skeleton, the craniofacial skeleton manifests many unique properties in response to external stimuli and signals. However, the mandibular or maxillary bone marrow mesenchyme, which is the intrinsic foundation of the functions of craniofacial skeleton, has not been well studied, and its homeostasis mechanism remains elusive. Osteoporosis is a systemic disease that affects all skeletons and is characterized by bone mass loss. Osteoporotic bone marrow mesenchymal stem cells (BMMSCs) exhibit disturbed homeostasis and distorted lineage commitment. Many reports have shown that microRNAs (miRNAs) play important roles in regulating MSCs homeostasis. Here, to obtain a better 
understanding of mandibular bone marrow MSCs homeostasis, we isolated and cultured mandible marrow MSCs from mouse mandibles. Using miR-705 mimics and an inhibitor, we demonstrated that miR-705 played a vital role in shifting the mandibular MSCs lineage commitment in vitro. Utilizing an osteoporosis mouse model, we demonstrated that MSCs from ovariectomized (OVX) mouse mandibular bone marrow exhibited impaired osteogenic and excessive adipogenic differentiation. miR-705 was found overexpressed in OVX mandibular MSCs. The knock down of miR-705 in vitro partially attenuated the differentiation disorder of the OVX mandibular MSCs by upregulating the expression of osteogenic marker genes but suppressing adipogenic genes. Taken together, our findings provide a better understanding of the homeostasis mechanism of mandibular BMMSCs and a novel potential therapeutic target for treating mandibular osteoporosis.

\section{Introduction}

Bone marrow homeostasis is vital for the function of bone marrow mesenchymal stem cells (BMMSCs) and thus the function of bones. Disturbance of homeostasis may result in a shift in cell lineage commitment of BMMSCs and cause systemic diseases, such as osteoporosis and hyperparathyroidism (Simonds et al. 2002). BMMSCs from osteoporotic bone marrow exhibit reduced osteogenic and enhanced adipogenic potential, resulting in constant bone mass loss and a decline in mechanical strength in all bones, including the craniofacial skeleton (Chen et al. 2016; Jonasson \& Rythen 2016; Mavropoulos et al. 2007). Despite 
49 many reports on this topic, the mechanisms behind the shift remain elusive.

Although the craniofacial, axial and appendicular skeletons share the same histological morphology, the craniofacial skeleton has a different homeostasis

52 mechanism than those of the axial and appendicular skeletons. Unlike the axial and

53 appendicular skeletons, the craniofacial skeleton is generated by neural crest cells

54 from the neuroectoderm germ layer (Li et al. 2017; Minoux \& Rijli 2010) and 55 undergoes intra-membranous ossification instead of endochondral ossification 56 (Minarikova et al. 2015). Differences can also be observed in the responses of 57 these skeletons to external stimuli. Systemic diseases, such as cherubism (Brix et al. 2009) and bisphosphonate-related osteonecrosis of the jaws (Dimitrakopoulos et al. 2006; Migliorati et al. 2005), affect only alveolar bones. Previous studies also showed that alveolar bones lost less trabecular bone mass at a lower rate in a

61 rodent osteoporosis model, which was quite different from the observations in long 62 bones (Mavropoulos et al. 2007). This evidence led us to hypothesize that MSCs in 63 the mandibular or maxillary bone marrow might possess some unique characters in 64 cell lineage commitment and homeostasis sustaining. In this study, we constructed 65 a mouse osteoporosis model to study the differentiation properties and regulatory mechanism of mandibular MSCs. We found that mandibular MSCs in the model

67 mouse exhibited lower osteogenic potential but higher adipogenic potential 68 through ex vivo induction and staining. RNA expression of marker genes provided 69 similar conclusions. Subsequently, we focused on microRNAs (miRNAs) to 
70 uncover the regulatory mechanism of mandibular MSCs.

miRNAs function by base pairing to complementary sites in the 3 ' untranslated region (3'UTR) of mRNAs (Ab Mutalib et al. 2016; Bartel 2009). The

73 connections between miRNAs and the cell lineage commitment of MSCs have 74 been demonstrated by several studies using BMMSCs from femurs (Chen et al. 75 2016). In our previous study, we identified miR-705 in mouse femur BMMSCs as 76 a negative regulator of osteoblast differentiation (Liao et al. 2013). Overexpression 77 of miR-705 caused extra bone mass loss in a mouse model, whereas its knockdown 78 attenuated bone loss in an ovariectomized (OVX) mouse model.

Here, based upon our previous discovery, we confirmed that miR-705 exerted a negative regulatory function in mouse mandibular MSCs. In the gain and loss 81 assay, we showed that miR-705 played a key role in regulating lineage 82 commitment in MSCs from OVX mouse mandibles. Knocking down miR-705 in

83 OVX mandibular MSCs resulted in elevated osteoblast differentiation but reduced adipocyte differentiation in vitro. Thus, our study suggested a novel regulatory mechanism in mandibular MSCs that might promote the discovery of new 86 therapeutic targets for osteoporotic alveolar bones. 
88

89

90

91

92

93

94

95

96

97

98

99

100

101

102

103

104

105

106

107

108

Materials and Methods

\section{Animals.}

Sixty 6-week-old female C57BL/6J mice were randomly divided into two groups. Mice in the Sham group underwent sham surgery. Mice in the OVX group received a bilateral OVX under general anaesthesia. After surgery, all mice were housed under pathogen-free conditions $\left(24^{\circ} \mathrm{C}, 12\right.$-hour light/12-hour dark cycles and $50-55 \%$ humidity) for 3 months. All animal procedures were performed according to the guidelines of the Animal Care Committee of Zunyi Medical University, Zunyi, Guizhou, China.

Cell culture.

Three months after the OVX and sham surgeries, MSCs were isolated from the mouse mandibles and cultured. Briefly, the mandibles were removed aseptically after euthanasia and dissected free of soft tissues. The periodontal tissue with the whole teeth was removed. Part of the alveolar bone surrounding the tooth socket was also removed to ensure clearance of dental pulp and odontoblast contamination. Then, the mandibles were smashed with scissors and flushed with $\alpha$-MEM (Invitrogen, Carlsbad, CA, USA, supplemented with 10\% FBS and 1\% penicillin and streptomycin) to free the marrow cavities. The cell suspension was filtered, seeded into 5-cm culture dishes and grown in $\alpha-M E M$ in a humidified atmosphere with $5 \% \mathrm{CO}_{2}$ at $37^{\circ} \mathrm{C}$. The medium was changed every two days, and adherent cells at confluence were passaged with $0.25 \%$ trypsin/ $1 \mathrm{mM}$ EDTA. Cells 
109 from passages 2-3 were used in the experiments.

110 Alizarin red staining.

111 Mandibular MSCs at the $2^{\text {nd }}$ passage were digested and seeded in osteogenesis112 inducing medium $(100 \mathrm{mg} / \mathrm{ml}$ ascorbic acid, $2 \mathrm{mM} \beta$-glycerophosphate and $10 \mathrm{nM}$

113 dexamethasone) for two weeks to induce osteoblast differentiation. Alizarin red 114 staining was performed as described previously (Yang et al. 2013). Quantification 115 analysis was performed by extracting mineralized nodules with cetylpyridinium 116 chloride and measuring the absorbance with a spectrophotometer at $570 \mathrm{~nm}$.

\section{Oil red O staining.}

118 Mandibular MSCs at the $2^{\text {nd }}$ passage were digested and seeded in adipogenesis119 inducing medium $(0.5 \mathrm{mM}$ isobutyl methylxanthine, $0.5 \mathrm{mM}$ dexamethasone and $12060 \mathrm{mM}$ indomethacin; Sigma-Aldrich, St. Louis, MO, USA) for one week for 121 adipogenic induction. Oil red O staining was performed to detect lipid droplets as 122 described previously (Yang et al. 2013). Quantification analysis was performed by 123 extracting lipid droplets with isopropanol and measuring the absorbance with a 124 spectrophotometer at $520 \mathrm{~nm}$.

125 Real-time $q$ RT-PCR analysis of mRNAs and miRNAs.

126 Total RNA was extracted using the TRIzol reagent (Invitrogen Life Technology, 127 Carlsbad, CA, USA). Single-strand cDNA was synthesized from $2 \mathrm{mg}$ of total 128 RNA with the PrimeScript RT reagent kit (TaKaRa Bio, Dalian, China). For the $129 q$ RT-PCR analysis, the miRNAs were reverse-transcribed with RT primers 
130 (RiboBio, Guangzhou, China). $\beta$-actin and U6 were used as loading controls for 131 quantitation of the mRNAs and miRNAs respectively. The Bulge-Loop miRNA $132 q$ RT-PCR Primer Set (RiboBio) was used for $q$ RT-PCR of miR-705 (Product ID: 133 miRQ0003495-1-2) and U6 (Product ID: MQP-0202). All real-time PCR analyses 134 were performed and analysed with the SYBR Premix Ex Taq II kit (TaKaRa) and 135 the ABI Prism 7500 HT sequence detection system (Applied Biosystems, Foster 136 City, CA, USA). The primer sequences are shown in Supplementary Table 1.

137

138 139

\section{Western blot.}

The western blot analyses were performed as previously described (Liao et al. 2013). The proteins were loaded on $10 \%$ sodium dodecyl sulphate polyacrylamide gels, transferred to polyvinylidene fluoride membranes (Millipore, Billerica, MA, USA) and blocked with $5 \%$ non-fat milk powder in PBST (containing $0.1 \%$ Tween). The membranes were probed overnight with the following primary antibodies: $\beta$-actin (Cell Signalling, Beverly, MA, USA), homeodomaincontaining factor A10 (HOXA10) (Santa Cruz, Dallas, TX, USA) and Forkhead box O1 (FoxO1) (Cell Signalling). The membranes were incubated with peroxidase-conjugated secondary antibody (Boster, Wuhan, China). The blots were visualized through an enhanced chemiluminescence kit (Amersham Biosciences, Piscataway, NJ, USA) according to the recommended instructions.

\section{Transfection of the miRNA mimics and inhibitor.}

The miR-705 mimics, inhibitor and negative control were purchased from RiboBio. 
151 The final concentration used for transfection was adjusted to $50 \mathrm{nM}$. The 152 siPORTNeoFX transfection reagent (Ambion, Austin, TX, USA) was used 153 according to the manufacturer's instruction. Briefly, diluted miRNA 154 mimics/inhibitor and diluted siPORTNeoFX transfection reagent were mixed and 155 incubated for $10 \mathrm{~min}$ at room temperature. Cultured cells at $80 \%$ confluence were 156 digested, and the suspensions were overlaid onto the transfection complexes and 157 incubated at $37^{\circ} \mathrm{C}$ for $48 \mathrm{~h}$. Control group cells received the transfection reagent 158 alone, and the other operations were the same.

159 Micro-CT analysis.

160 The mice were scanned with the Inveon micro-CT system (Siemens AG, Germany). 161 The scans were performed using an $80-\mathrm{kV}$ and $500-\mathrm{mA}$ micro-focus X-ray source. 162 The cross-sectional volumetric bone mineral density (BMD) was measured at the 163 angle of the mandible (the rectangle area near the joint area of the body and the 164 ramus of the mandible posterior to the molars). Bone morphometric parameters 165 and the bone volume relative to the tissue volume (BV/TV) were also assessed. 166 Micro-CT scanning of femurs was performed as described previously (Yang et al. 167 2013).

168 Statistical analysis.

169 The data are presented as the means \pm SDs. Comparisons were made using one170 way ANOVA followed by Tukey-Kramer test as a post-hoc. All experiments were 171 repeated at least three times, and representative experiments were shown. 
172 Differences were considered significant at $P<0.05$.

173

174 
175

176

177

178

179

180

181

182

183

184

185

186

187

188

189

190

191

192

193

194

195

\section{Results}

Cell lineage commitment of mandibular MSCs shifts to adipocytes during osteoporosis.

The OVX models were constructed and confirmed as previously described (Liao et al. 2013). As expected, all mice manifested osteoporosis 3 months after OVX. In addition to the femur results, we also focused on the mandible (Fig. 1a). The BMD and BV/TV were significantly reduced in the femurs of OVX mouse (Figs. 1b, 1c). However, the BMD and BV/TV comparisons were barely significant between the OVX and Sham mandibles, which were quite different from the results obtained in the long bones (Figs. 1d, 1e).

MSCs were isolated from both the OVX and Sham mouse mandibles and cultured in vitro for further investigation. The flow cytometry analysis showed that these cells were all positive for CD29, CD105, CD106 and SCA-1 but were negative for CD34 and CD45. No significant difference was observed in the proliferation comparison.

We then compared the differentiation potentials of the OVX and Sham mandibular MSCs. The OVX mandibular MSCs formed much fewer mineralized nodules but more adipocytes than the Sham MSCs during induction, suggesting that weaker osteogenic but stronger adipogenic differentiation occurred in the OVX mandibular MSCs (Figs. 2a, 2b, 2e and 2f). This result was confirmed by $q$ RT-PCR. Runt-related transcription factor 2 (RUNX2) and alkaline phosphatase 
196 (ALP) were analysed as two osteogenic marker genes. The OVX mandibular 197 MSCs expressed significantly less RUNX2 and ALP than the Sham MSCs after 2 198 weeks of osteogenic induction (Figs. 2c, 2d). However, after 1 week of adipogenic 199 induction, the expression of peroxisome proliferator-activated receptor- $\gamma$ (PPAR- $\gamma$ ) 200 and lipoprotein lipase (LPL), which were adipogenic marker genes, were 201 significantly increased compared with the levels in the Sham MSCs (Figs. 2g, 2h).

202

203

204

205

206

207

208

209

210

211

212

213

214

215

216

\section{Osteoporotic mandibular MSCs overexpress miR-705.}

In a previous study, we detected the overexpression of miR-705 in osteoporotic bone marrow using MSCs from OVX mouse femurs. Here, we confirmed that miR-705 was also overexpressed in osteoporotic mouse mandible BMMSCs. The $q$ RT-PCR results showed that miR-705 was significantly upregulated in $3^{\text {rd }}$-passage OVX mouse mandibular MSCs compared to the expression level in the Sham MSCs (Fig. 3a).

Subsequently, to evaluate whether miR-705 affected osteogenic differentiation in mandibular MSCs, we examined miR-705 expression during osteogenic induction in vitro. According to the $q$ RT-PCR results, miR-705 expression declined during osteogenic induction, with minimum expression witnessed on day 7 (Fig. 3b). Conversely, its expression increased during adipogenic induction and hit the maximum level on day 7 (Fig. 3c).

Inhibition of MSC osteoblast lineage commitment by miR-705.

To investigate its role in cell lineage commitment, we knocked down and 
217 overexpressed miR-705 in mandibular MSCs with the si-miR-705 (inhibitor) and 218 mimics respectively. The effects of the miRNA inhibitor and mimics were 219 confirmed by $q$ RT-PCR up to day 14 of induction (Supplementary Fig. 1a). 220 Alizarin red staining after 14 days of induction confirmed enhanced nodule 221 formation by mandibular MSCs after miR-705 knockdown (Figs. 4a-4d). And $222 q$ RT-PCR showed enhanced expression of both ALP and RUNX2 in the 223 knockdown MSCs (Figs. 4e, 4f). However, overexpression of miR-705 by the 224 mimics significantly decreased mineralization of the mandibular MSCs as well as 225 the ALP and RUNX2 expression levels.

226

227

228

229

230

231

232

233

234

235

237

236 Knockdown of miR-705 rescues the cell lineage commitment disorder of

Promotion of MSC adipocyte lineage commitment by miR-705.

To assess the function of miR-705 in adipocyte lineage commitment, we conducted a gain or loss of function assay on the mandibular MSCs. The effects of the miRNA inhibitor and mimics were confirmed by $q$ RT-PCR up to day 7 of induction (Supplementary Fig. 1b). Oil red O staining showed fewer fat droplets after 7 days of adipo-induction in the miR-705 knockdown MSCs (Figs. 5a-5d). Expression of the adipogenic marker genes LPL and PPAR- $\gamma$ was also decreased on day 7 in the $q$ RT-PCR assay (Figs. 5e, 5f). Conversely, promotion of both adipocyte formation and LPL and PPAR- $\gamma$ expression was observed in the miR705-overexpressing MSCs. 37 MSCs from osteoporotic mandibles. 
Several miRNAs were shown to have functions in shifting MSC linage 239 commitment. In this study, we tested whether knocking down miR-705 attenuated 240 the lineage commitment disorder in MSCs from osteoporotic mandibles. Two 241 groups of OVX mandibular MSCs were transfected either with the miR-705 242 inhibitor or the transfection reagent alone. And a group of Sham mandibular MSCs

243 served as the control. As shown in Fig. 6, knocking down miR-705 partially 244 rescued osteogenic differentiation of the OVX mandibular MSCs after osteo245 induction. Significantly more mineralized nodules were observed in the 246 knockdown group than those in the OVX group (Figs. 6a-6d). This result was 247 confirmed by $q$ RT-PCR, which showed increased RUNX2 and ALP mRNA 248 expression in the OVX mandibular MSCs after miR-705 knockdown (Figs. 6e, 6f). 249 Next, we tested whether the knockdown of miR-705 reduced the excessive 250 adipogenic differentiation of the OVX mandibular MSCs in the same way. The 251 knockdown of miR-705 resulted in reduced lipid droplet accumulation in the 252 induced OVX mandibular MSCs to a certain extent (Figs. 7a-7d). Consistently, it 253 reduced the expression of the adipogenic marker PPAR- $\gamma$ and LPL (Figs. 7e, 7f). 254 These results suggested that the knockdown of miR-705 in OVX mandibular 255 MSCs partially attenuated their adipogenic differentiation. 
To uncover details of the attenuation observed above, we tested the

259

260

261

262

263

264

265

266

267

268

269

270

271

272

273

274

275

276

277

278

transcription levels of its predicted target mRNAs HOXA10 and FoxO1 at $48 \mathrm{~h}$

post-transfection. As shown in the $q \mathrm{RT}$-PCR results, miR-705 expression was remarkably reduced at $48 \mathrm{~h}$ after inhibitor transfection (Fig. 8a). According to western blot results, HOXA10 and FoxO1 were suppressed in the MSCs from the OVX mandibles compared with the negative controls but were increased significantly 2 days after miR-705 inhibitor transfection (Fig. 8b).

\section{Discussion}

In this study, we cultured MSCs from osteoporotic mouse mandibles and demonstrated that miR-705 acted negatively in mandibular MSC osteoblast but positively in adipocyte lineage commitment. Additionally, inhibiting miR-705 in osteoporotic mandibular MSCs reduced adipogenic differentiation and attenuated the cell lineage commitment disorder.

Studies have reported that systemic diseases, such as osteoporosis and Paget's disease, affect all bones, including alveolar bones (Galson \& Roodman 2014). Although the oestrogen deficiency caused by ovariectomy leads to bone mass loss in both craniofacial and long bones, the mandible loses significantly less bone mass than the proximal tibia according to reports from SD rats (Mavropoulos et al. 2007). However, some diseases, such as cherubism (Brix et al. 2009) and bisphosphonate-related osteonecrosis of the jaws (Dimitrakopoulos et al. 2006; Migliorati et al. 2005), are only witnessed in the craniofacial skeleton. Their 
279 distinct responses to external stimuli imply that mandibles may have a different

280 homeostasis mechanism than long bones. Since a disturbance of the alveolar bone

281 marrow may cause many problems to stomatological treatment such as

282 prosthodontics and maxillofacial surgery (Chen et al. 2016), obtaining a better

283 understanding of homeostasis in mandibular MSCs will help improve treatment of

284 these diseases.

285 In fact, the craniofacial, axial and appendicular skeletons have different 286 developmental origins. The craniofacial structures are primarily formed by neural 287 crest cells, which migrate to the branchial arches during the development process 288 and undergo intra-membranous ossification (Li et al. 2017; Minarikova et al. 2015; 289 Minoux \& Rijli 2010). In contrast, the axial and appendicular skeleton originates 290 from mesenchymal condensations of the mesoderm and adopts endochondral 291 ossification during development (Kawata et al. 2017; Olivares-Navarrete et al. 292 2017). All of this evidence suggests that the MSCs in the mandible may play 293 different roles than their counterparts in the long bone marrow.

294 In this study, we established an osteoporosis model in mice and isolated 295 MSCs from the mandibular bone marrow. To rule out interference of dental pulp 296 and odontoblasts, the periodontal tissue with the whole teeth was removed. Unlike 297 human molars, mouse molars keep erupting for their entire lifespan, thus the force 298 generated during use of the alveolar bone beneath the molars keeps changing. To 299 avoid this interference, we chose the rectangular area posterior to the molar as the 
300 301 human mandible. As expected, the BMD and BV/TV comparisons were barely

302 significant between the OVX and Sham mouse mandibles, which was quite 303 different from the results obtained from the femur comparisons. The distinct 304 mechanical loading on the mandible may play a role in this protective effect 305 (Mavropoulos et al. 2014; Mavropoulos et al. 2010; Mavropoulos et al. 2007). 306 307 308 309 310 311 312 313 314 315 316 317 318 319 detailed differences. miRNAs regulate MSC differentiation through the 320

scan target for micro-CT, which was equivalent to the angulus mandibulae in the Previous studies have shown that mechanical loading on long bones is quite different from that on the mandible and that the mandible is less sensitive to protein under-nutrition or OVX than the proximal tibia spongiosa (Mavropoulos et al. 2014; Mavropoulos et al. 2010). However, these phenomena were not seen in the MSCs isolated from the OVX mandibular bone marrow which, like the MSCs in the OVX long bones, exhibited weaker osteogenic but stronger adipogenic potential in vitro. Analysis of marker gene expression gave similar results. RUNX2 and ALP were expressed at lower levels, but PPAR- $\gamma$ and LPL were expressed at higher levels in the OVX mandibular MSCs than those in the Sham MSCs. The key to the less-sensitive character of the mandible in vivo might lay in the unique microenvironment of mandibular MSCs. Therefore we started with the known clue to identify the main factor in the microenvironment. We focused on miRNAs that were differently expressed in OVX and Sham MSCs in an attempt to unveil the downregulation of target gene expression by either mRNA degradation or 
321 translational inhibition (Ab Mutalib et al. 2016; Bartel 2009; Yang et al. 2017).

322 Their ability to regulate BMMSC lineage commitment has drawn much attention.

$323 \mathrm{miR}-21, \mathrm{miR}-22$, miR-27a, and miR-183 have all been reported to regulate the

324 differentiation of BMMSCs (Gong et al. 2016; Ke et al. 2015; Yan et al. 2017;

325 Zheng et al. 2017). In a previous study, we demonstrated a regulatory role of miR-

326705 and miR-3077 in the aetiology of postmenopausal osteoporosis in long bones

327 (Liao et al. 2013). Here, we focused on miR-705 to determine whether it regulated

328 mandibular bone marrow MSCs. We found that miR-705 was overexpressed in the

329 MSCs isolated from osteoporotic mouse mandibles. Upregulating miR-705 in

330 mandibular MSCs with mimics resulted in less abundant mineral nodules during

331 osteogenic induction but more lipid droplets during adipogenic induction.

332 Downregulating it with an inhibitor gave opposite outcomes. $q$ RT-PCR analysis of

333 both osteogenic and adipogenic marker genes confirmed these results. Thus we

334 hypothesized that miR-705 played a crucial role in mandibular MSC lineage

335 commitment and mandibular bone marrow homeostasis.

336 Next, we used the OVX mandibular MSCs to further investigate the role of 337 miR-705 in regulating the homeostasis of these cells. Transfection of the miR-705

338 inhibitor into OVX mandibular MSCs partially attenuated the impaired osteogenic

339 potential and suppressed the excessive adipogenic differentiation to a certain extent. 340

These results confirmed our hypothesis that miR-705 inhibited osteoblast 
342 differentiation and promoted adipocyte differentiation of mandibular MSCs.

343 To investigate the mechanism described above, we tested the mRNA 344 expression levels of HOXA10 and FoxO1, which are two important osteogenic 345 genes and predicted targets of miR-705. Previously, we demonstrated that 346 overexpression of miR-705 suppressed HOXA10 and FoxO1 expression and that 347 they could be partially restored by miR-705 inhibitor transfection (Liao et al. 2016; 348 Liao et al. 2013). Here we found that miR-705 exerted similarly in OVX 349 mandibular MSCs and that its inhibitor increased HOXA10 and FoxO1 protein 350 expression to a certain extent. However, with numerous predicted target mRNAs, 351 we cannot conclude that miR-705 has the same effects in the mandible and long 352 bones. The mechanism underlying this finding requires more work in the future.

353 Taken together, our results showed that miR-705 was overexpressed in 354 osteoporotic mandibular MSCs and functioned as a negative regulator of osteoblast 355 differentiation. Suppression of the elevated miRNA by synthetic oligonucleotides 356 may partially rescue the lineage commitment disorder of OVX mandibular MSCs. 357 Utilizing an OVX mouse model, we provided a better understanding of the 358 mechanisms underlying mandibular MSC homeostasis and thus identified a 359 potential therapeutic target for the treatment of mandibular osteoporosis. 360 CONCLUSIONS 
Ab Mutalib NS, Othman SN, Mohamad Yusof A, Abdullah Suhaimi SN, Muhammad R, and Jamal R. 2016. Integrated microRNA, gene expression and transcription factors signature in papillary thyroid cancer with lymph node metastasis. PeerJ 4:e2119. 10.7717/peerj.2119

Bartel DP. 2009. MicroRNAs: target recognition and regulatory functions. Cell 136:215-233. 10.1016/j.cell.2009.01.002

Brix M, Peters H, and Lebeau J. 2009. [Cherubism]. Rev Stomatol Chir Maxillofac 110:293-298. 10.1016/j.stomax.2009.09.006

Chen N, Sui BD, Hu CH, Cao J, Zheng CX, Hou R, Yang ZK, Zhao P, Chen Q, Yang QJ, Jin Y, and Jin F. 2016. microRNA-21 Contributes to Orthodontic Tooth Movement. I Dent Res 95:1425-1433. $10.1177 / 0022034516657043$

Dimitrakopoulos I, Magopoulos C, and Karakasis D. 2006. Bisphosphonate-induced avascular osteonecrosis of the jaws: a clinical report of 11 cases. Int J Oral Maxillofac Surg 35:588-593. 10.1016/j.ijom.2006.02.022

Galson DL, and Roodman GD. 2014. Pathobiology of Paget's Disease of Bone. J Bone Metab 21:85-98. 10.11005/jbm.2014.21.2.85

Gong Y, Lu J, Yu X, and Yu Y. 2016. Expression of Sp7 in Satb2-induced osteogenic differentiation of mouse bone marrow stromal cells is regulated by microRNA-27a. Mol Cell Biochem 417:7-16. 10.1007/s11010-0162709-y

Jonasson G, and Rythen M. 2016. Alveolar bone loss in osteoporosis: a loaded and cellular affair? Clin Cosmet Investig Dent 8:95-103. 10.2147/CCIDE.S92774

Kawata K, Kubota S, Eguchi T, Aoyama E, Moritani NH, Oka M, Kawaki H, and Takigawa M. 2017. A Tumor Suppressor Gene Product, Platelet-Derived Growth Factor Receptor-Like Protein Controls Chondrocyte Proliferation and Differentiation. J Cell Biochem 118:4033-4044. 10.1002/jcb.26059

Ke K, Sul OJ, Rajasekaran M, and Choi HS. 2015. MicroRNA-183 increases osteoclastogenesis by repressing heme oxygenase-1. Bone 81:237-246. 10.1016/j.bone.2015.07.006

Li J, Parada C, and Chai Y. 2017. Cellular and molecular mechanisms of tooth root development. Development 144:374-384. 10.1242/dev.137216

Liao L, Yang X, Su X, Hu C, Zhu X, Yang N, Chen X, Shi S, Shi S, and Jin Y. 2013. Redundant miR-3077-5p and miR705 mediate the shift of mesenchymal stem cell lineage commitment to adipocyte in osteoporosis bone marrow. Cell Death Dis 4:e600. 10.1038/cddis.2013.130

Mavropoulos A, Kiliaridis S, Rizzoli R, and Ammann P. 2014. Normal masticatory function partially protects the rat mandibular bone from estrogen-deficiency induced osteoporosis. J Biomech 47:2666-2671. 10.1016/j.jbiomech.2014.05.012

Mavropoulos A, Odman A, Ammann P, and Kiliaridis S. 2010. Rehabilitation of masticatory function improves the 
alveolar bone architecture of the mandible in adult rats. Bone 47:687-692. 10.1016/j.bone.2010.06.025

Mavropoulos A, Rizzoli R, and Ammann P. 2007. Different responsiveness of alveolar and tibial bone to bone loss stimuli. J Bone Miner Res 22:403-410. 10.1359/jbmr.061208

Migliorati CA, Schubert MM, Peterson DE, and Seneda LM. 2005. Bisphosphonate-associated osteonecrosis of mandibular and maxillary bone: an emerging oral complication of supportive cancer therapy. Cancer 104:83-93. 10.1002/cncr.21130

Minarikova M, Oralova V, Vesela B, Radlanski RJ, and Matalova E. 2015. Osteogenic Profile of Mesenchymal Cell Populations Contributing to Alveolar Bone Formation. Cells Tissues Organs 200:339-348. 10.1159/000439165

Minoux M, and Rijli FM. 2010. Molecular mechanisms of cranial neural crest cell migration and patterning in craniofacial development. Development 137:2605-2621. 10.1242/dev.040048

Olivares-Navarrete R, Lee EM, Smith K, Hyzy SL, Doroudi M, Williams JK, Gall K, Boyan BD, and Schwartz Z. 2017. Substrate Stiffness Controls Osteoblastic and Chondrocytic Differentiation of Mesenchymal Stem Cells without Exogenous Stimuli. PLoS One 12:e0170312. 10.1371/journal.pone.0170312

Simonds WF, James-Newton LA, Agarwal SK, Yang B, Skarulis MC, Hendy GN, and Marx SJ. 2002. Familial isolated hyperparathyroidism: clinical and genetic characteristics of 36 kindreds. Medicine (Baltimore) 81:1-26.

Yan GQ, Wang X, Yang F, Yang ML, Zhang GR, Wang GK, and Zhou Q. 2017. MicroRNA-22 Promoted Osteogenic Differentiation of Human Periodontal Ligament Stem Cells by Targeting HDAC6. J Cell Biochem 118:16531658. 10.1002/jcb.25931

Yang N, Wang G, Hu C, Shi Y, Liao L, Shi S, Cai Y, Cheng S, Wang X, Liu Y, Tang L, Ding Y, and Jin Y. 2013. Tumor necrosis factor alpha suppresses the mesenchymal stem cell osteogenesis promoter miR-21 in estrogen deficiency-induced osteoporosis. J Bone Miner Res 28:559-573. 10.1002/jbmr.1798

Yang S, Sui J, and Liang G. 2017. Diagnosis value of aberrantly expressed microRNA profiles in lung squamous cell carcinoma: a study based on the Cancer Genome Atlas. PeerJ 5:e4101. 10.7717/peerj.4101

Zheng Y, Liu L, Chen C, Ming P, Huang Q, Li C, Cao D, Xu X, and Ge W. 2017. The extracellular vesicles secreted by lung cancer cells in radiation therapy promote endothelial cell angiogenesis by transferring miR-23a. PeerJ 5:e3627. 10.7717/peerj.3627 


\section{Figure 1}

Micro-CT and quantification of mouse mandible and femur.

(a) Micro-CT analysis of mandibles from the Sham and OVX mice. The red area is the target area for quantification. (b) BMD and (c) BV/TV comparisons of femurs from the Sham and OVX mice. (d) BMD and (e) BV/TV comparisons of mandibles from the Sham and OVX mice. Values are described as the mean \pm SD from three independent experiments. $* P<0.05, * * P$ $<0.01$; Sham, sham surgery; OVX, ovariectomy; BMD, bone mineral density; BV/TV, bone volume/total volume.
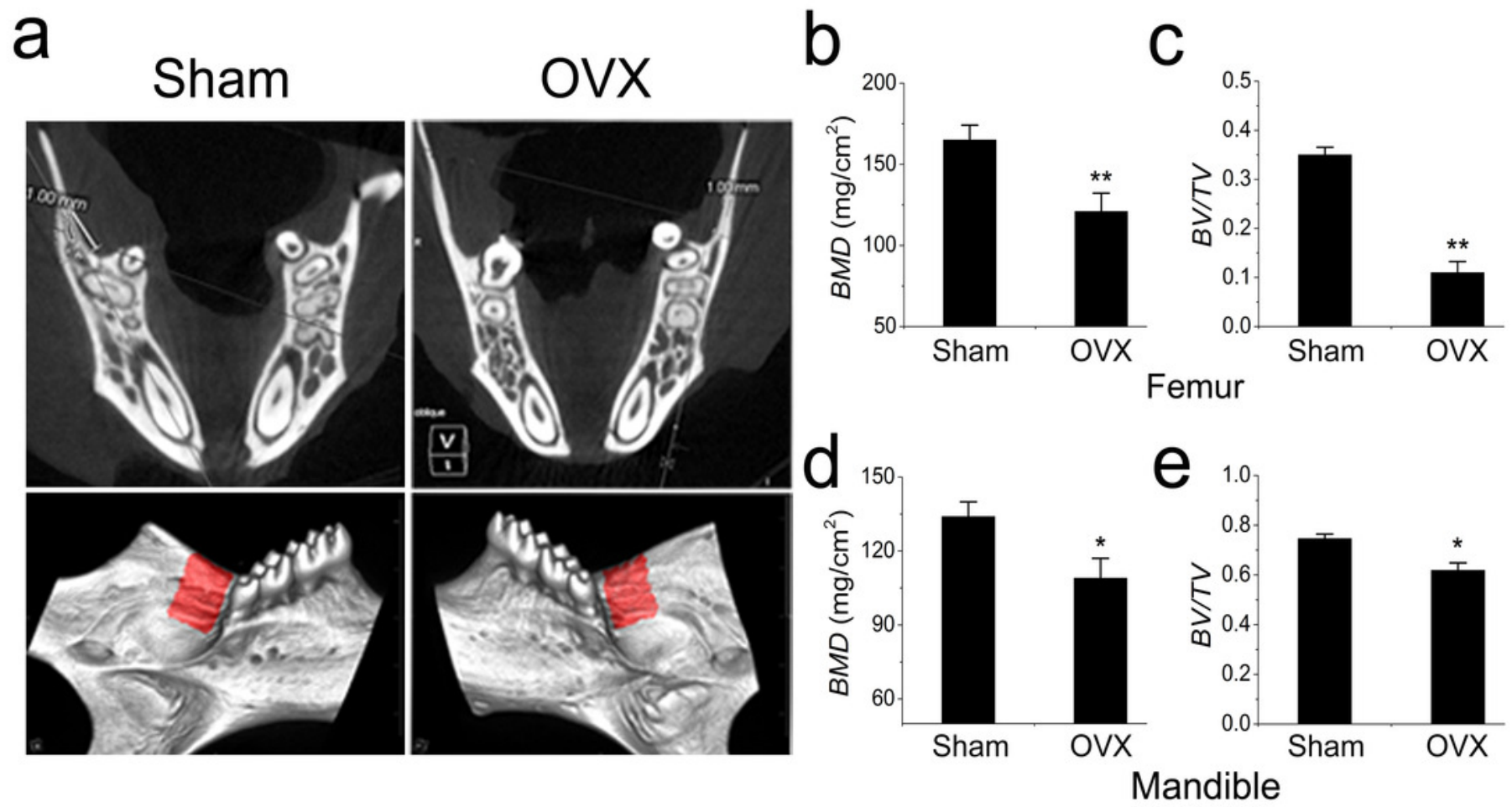


\section{Figure 2}

Shift of cell lineage commitment in MSCs from osteoporosis mandible bone marrow.

Osteoblast differentiation was impaired in MSCs from osteoporosis mandible bone marrow. MSCs from OVX and Sham mandible at P3 were induced with osteogenic medium for 14 days. Alizarin red staining was performed (a) and quantified (b) via extraction with cetylpyridinium chloride. qRT-PCR was used to determine the expression of RUNX2 (c) and ALP (d) in P3 MSCs. Adipocyte differentiation was enhanced in MSCs from osteoporosis mandible. MSCs from OVX and Sham mandible at P3 were induced with adipogenic medium for 7 days. Oil red O staining was performed (e) and quantified (f) via extraction with isopropanol. qRT-PCR was used to determine the expression of PPAR-y $(\mathbf{g})$ and LPL $(\mathbf{h})$ in P3 MSCs. The scale bars in micrographs represent $50 \mu \mathrm{m}$. Values are described as mean \pm SD from three independent experiments. $* P<0.05, * * P<0.01$. P3, third passage.
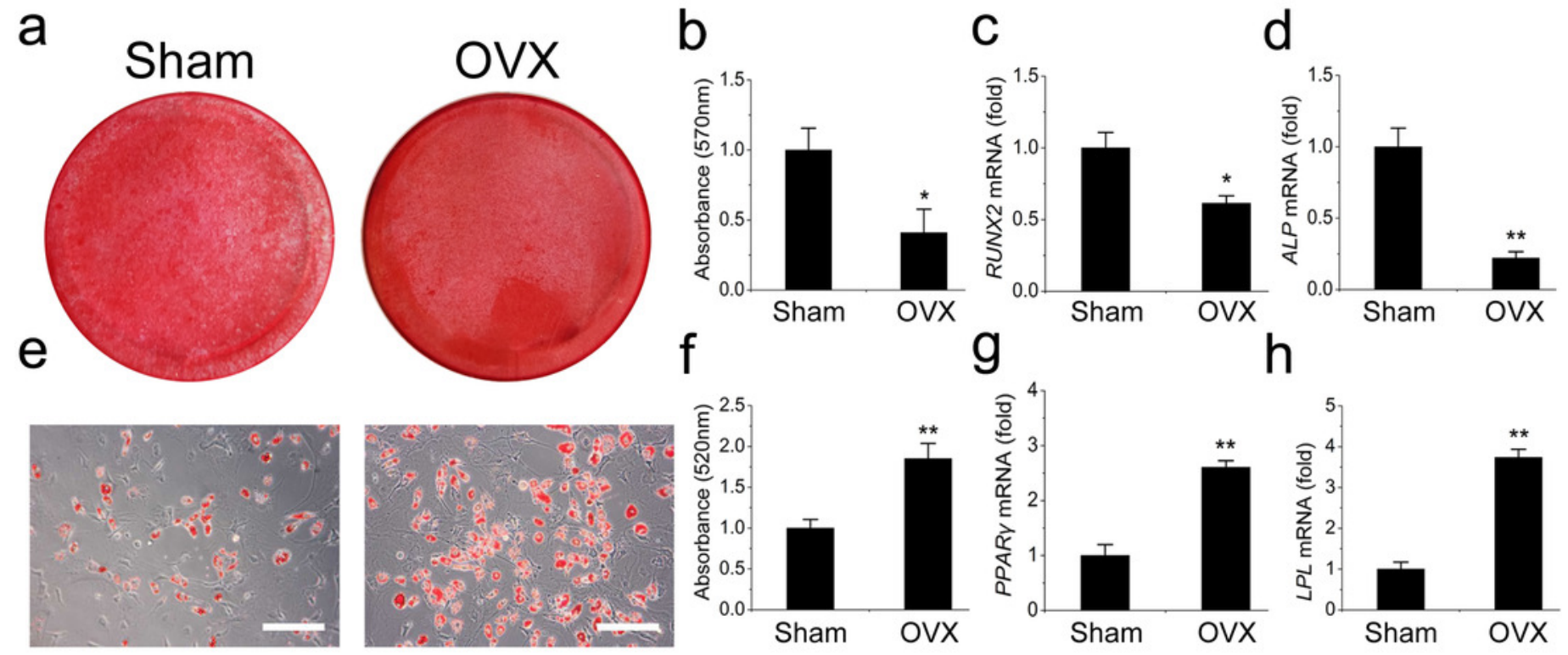


\section{Figure 3}

miR-705 is enhanced in MSCs from osteoporosis bone marrow.

qRT-PCR was used to measure the levels of miR-705 in the third passage of MSCs (a) and at different time point of osteogenic induction (b) and adipogenic induction (c). Values are described as mean \pm SD from three independent experiments. $* P<0.01,{ }^{* *} P<0.001$.
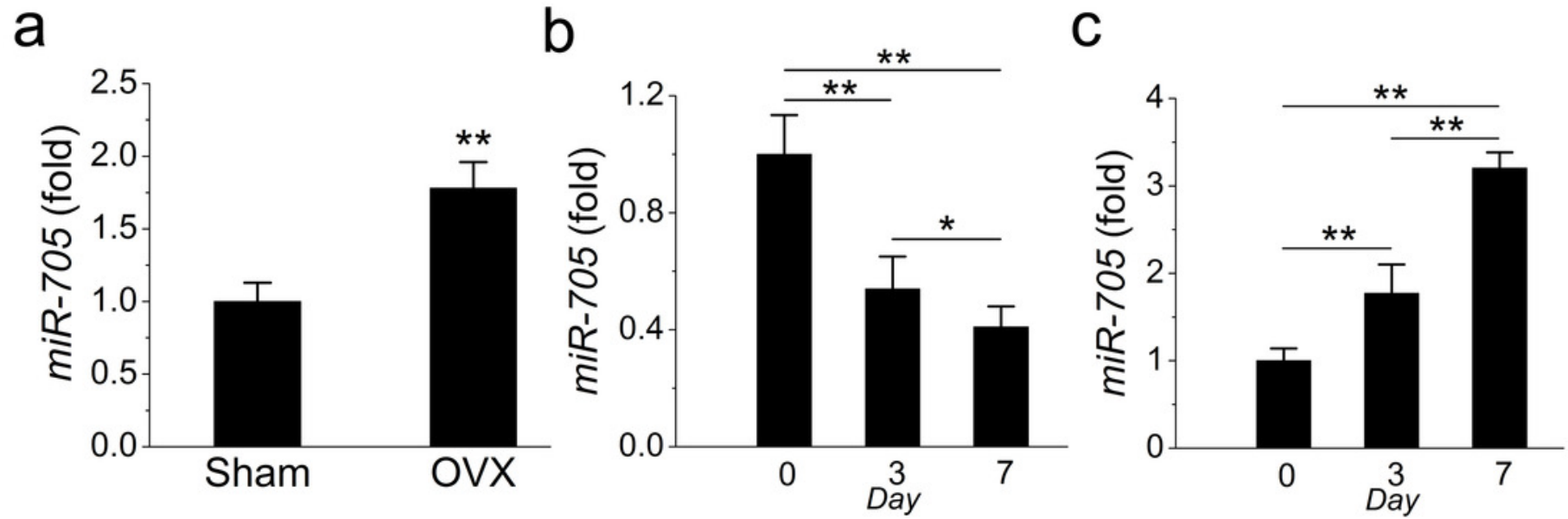


\section{Figure 4}

miR-705 inhibits osteoblast differentiation of MSCs.

MSCs were transfected with miR-705 mimics, inhibitor and the reagent as a negative control. Two days post-transfection, the cells were induced with osteogenic medium for 14 days. After osteogenic induction, alizarin red staining was performed (a-c) and quantified (d). ALP (e) and RUNX2 (f) expression in three groups was measured by qRT-PCR. The scale bars in the micrographs represent $100 \mu \mathrm{m}$. Values are described as the mean \pm SD from three independent experiments. $* P<0.01, * * P<0.001$.

a Control/MSC
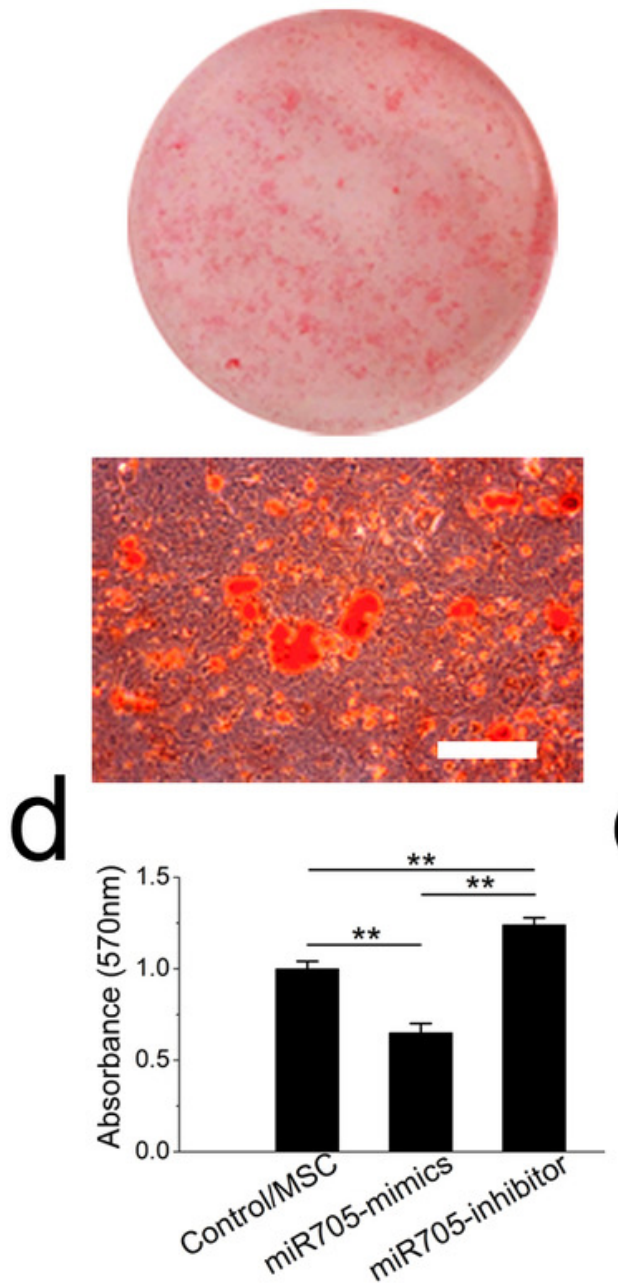

$b$
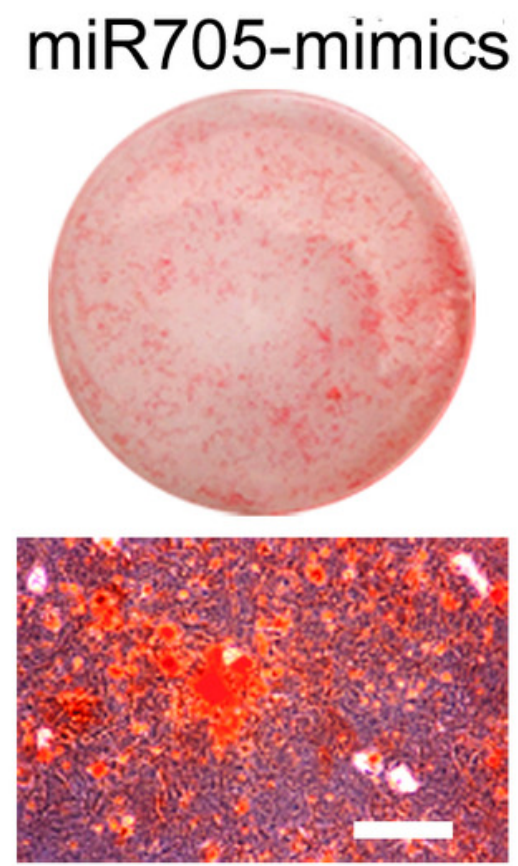

e

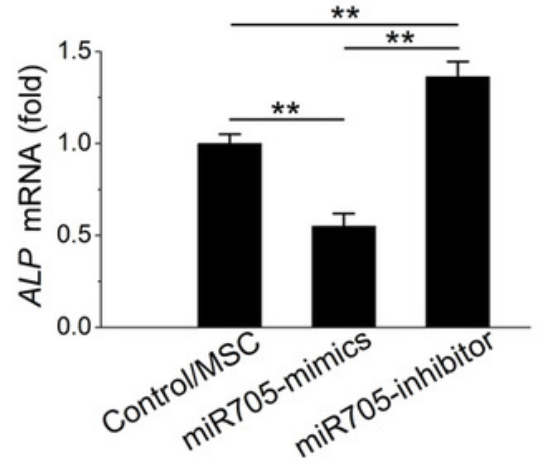

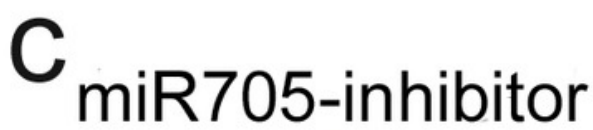
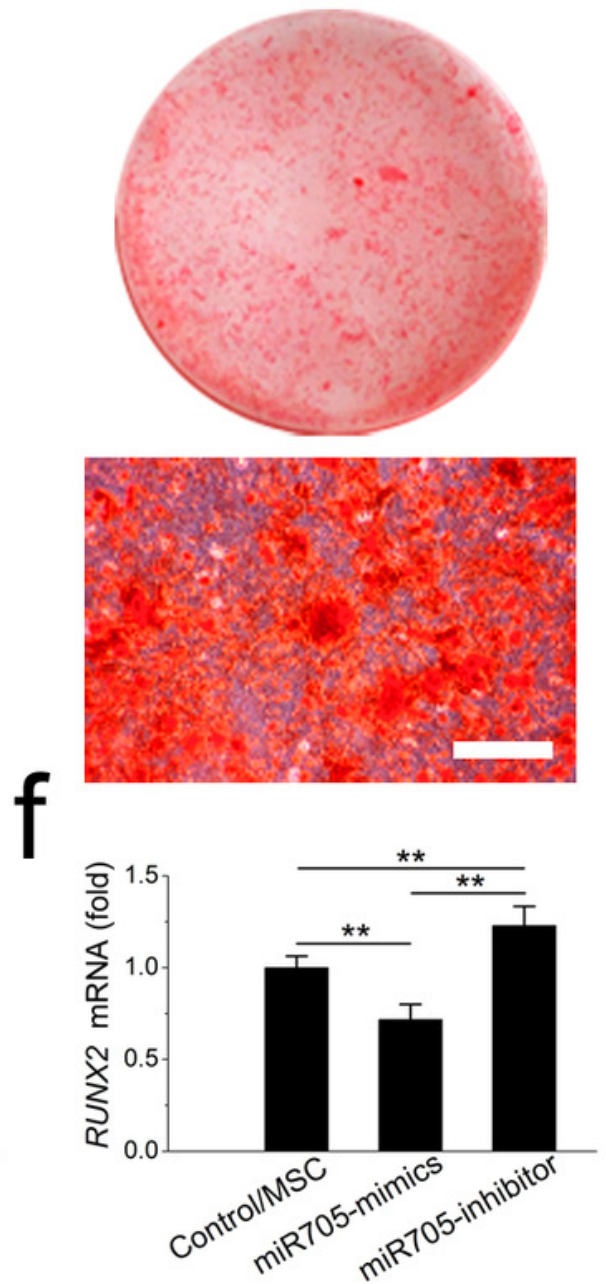


\section{Figure 5}

miR-705 promotes adipocyte differentiation of MSCs.

MSCs were transfected with miR-705 mimics, inhibitor and the reagent as a negative control. Two days post-transfection, the cells were induced with adipogenic medium for 7 days. After adipogenic induction, oil red $\mathrm{O}$ staining was performed (a-c) and quantified (d). LPL (e) and PPAR-y (f) expression in three groups was measured by qRT-PCR. The scale bars in the micrographs represent $100 \mu \mathrm{m}$. Values are described as the mean \pm SD from three independent experiments. $* P<0.01, * * P<0.001$.

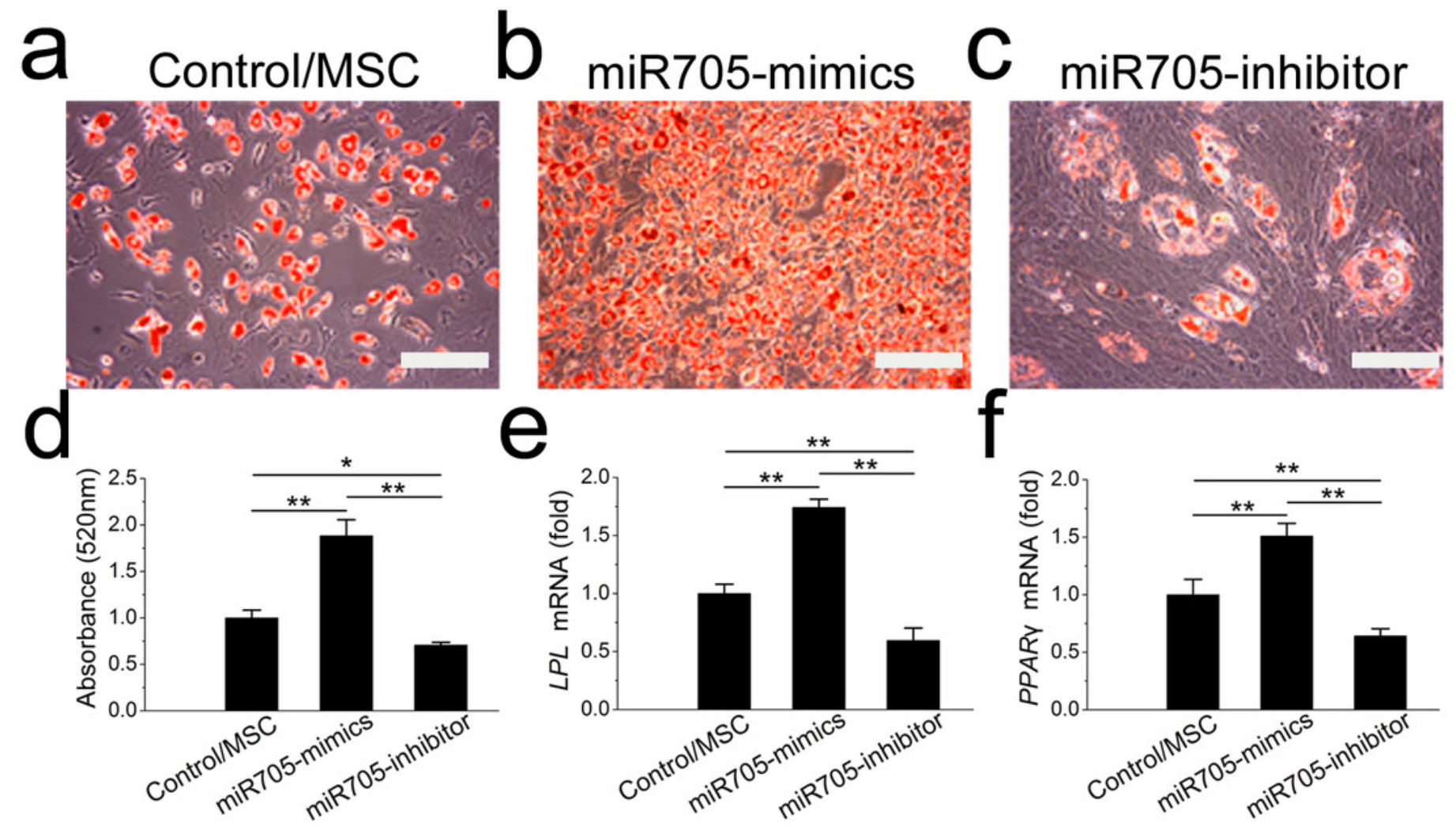




\section{Figure 6}

Knockdown of miR-705 promotes osteogenic differentiation of OVX MSCs.

Sham MSCs were transfected with the reagent as a negative control. OVX MSCs were transfected with the reagent and miR-705 inhibitor. Three group MSCs were cultured with osteogenic medium for 14 days. After osteogenic induction, alizarin red staining was performed (a-c) and quantified (d). RUNX2 (e) and ALP (f) expression in three groups was measured by $q \mathrm{RT}$-PCR. The scale bars in the micrographs represent $100 \mu \mathrm{m}$. Values are described as the mean \pm SD from three independent experiments. $* P<0.01,{ }^{* *} P<0.001$.

a

Control/Sham

b

C

Control/OVX OVX/miR705-inhibitor
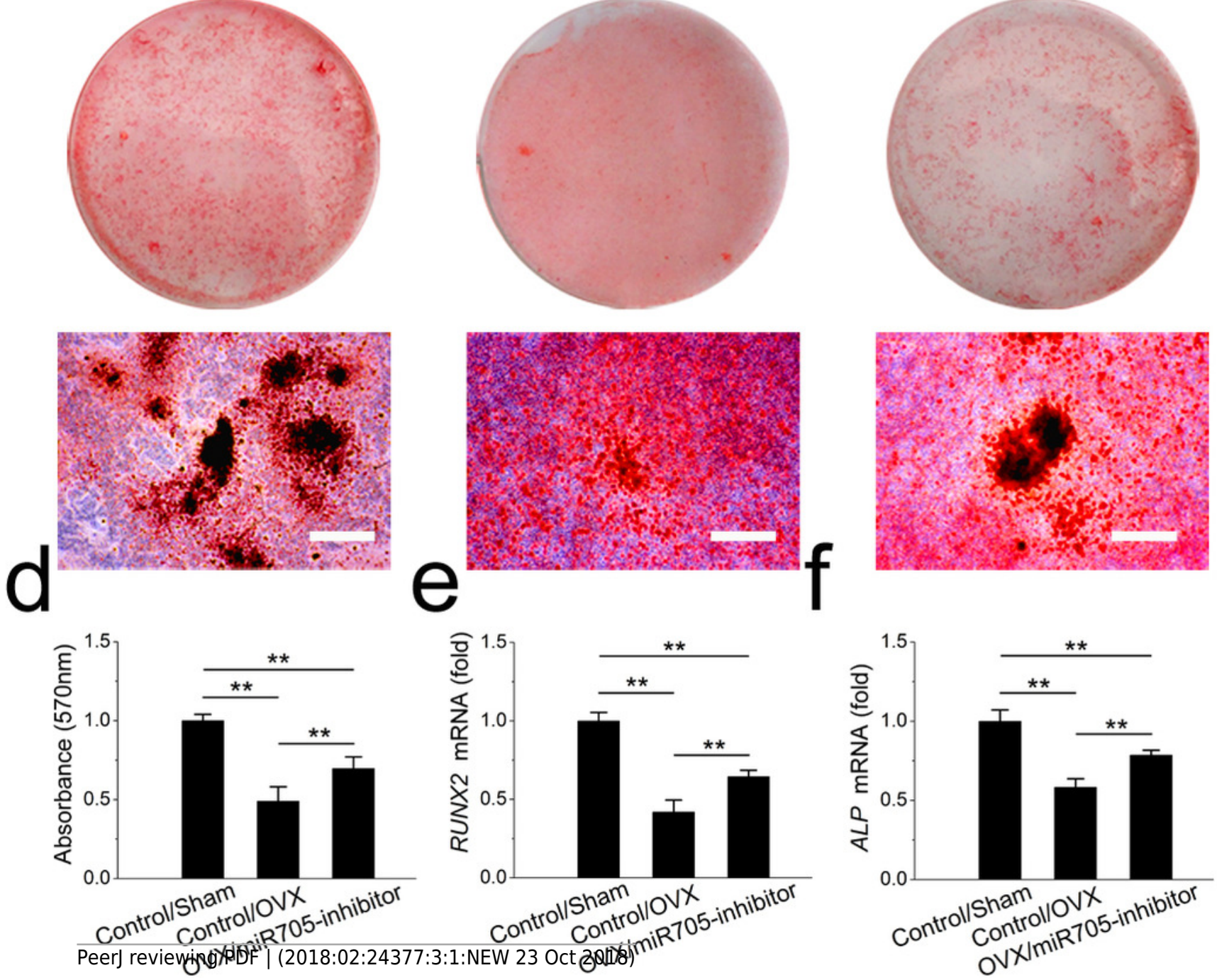


\section{Figure 7}

Knockdown of miR-705 inhibits adipogenic differentiation of OVX MSCs.

Sham MSCs were transfected with the reagent as a negative control. OVX MSCs were transfected with the reagent and miR-705 inhibitor. Three group MSCs were cultured with adipogenic medium for 7 days. After adipogenic induction, oil red $\mathrm{O}$ staining was performed (a-c) and quantified (d). PPAR-y (e) and LPL (f) expression in three groups was measured by qRT-PCR. The scale bars in the micrographs represent $50 \mu \mathrm{m}$. Values are described as the mean \pm SD from three independent experiments. $* P<0.01, * * P<0.001$.

a

Control/Sham

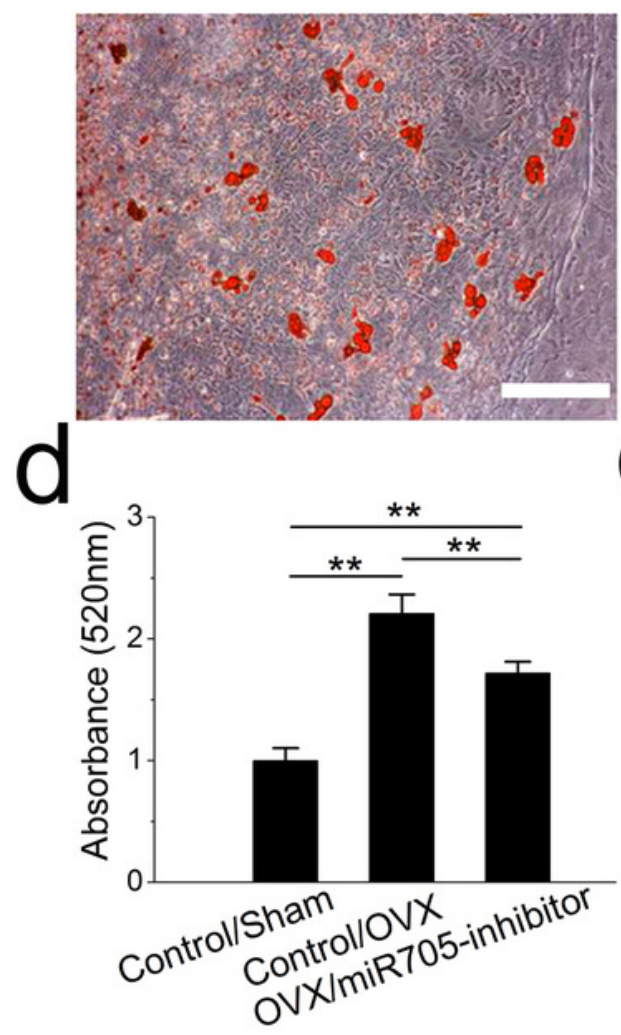

C

b
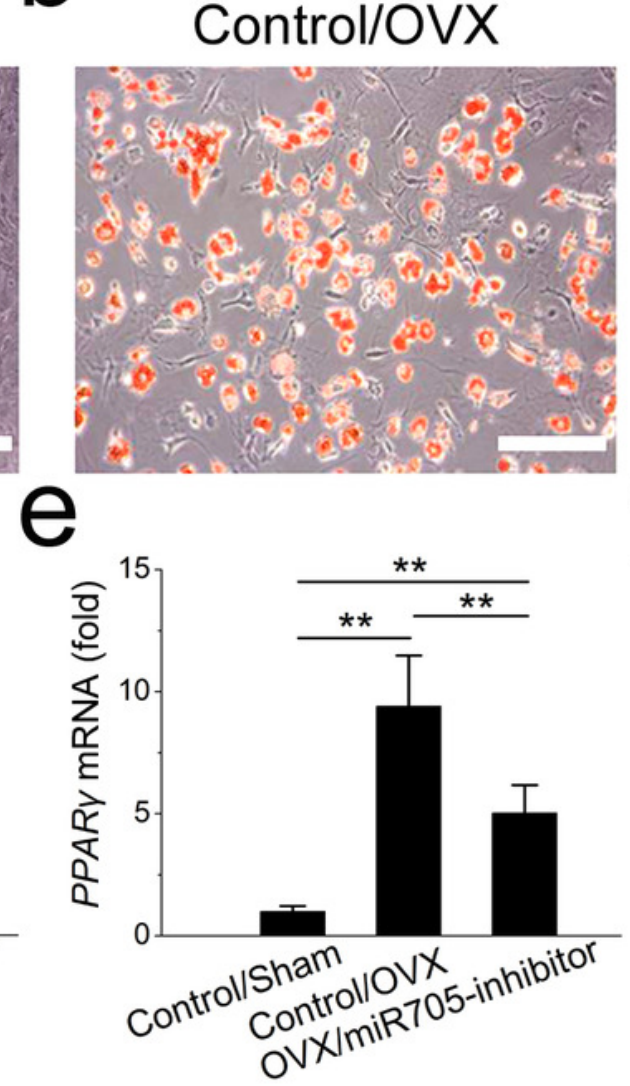

Control/OVX

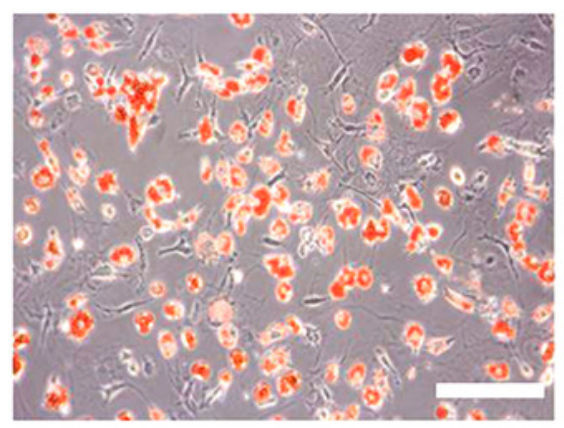

f

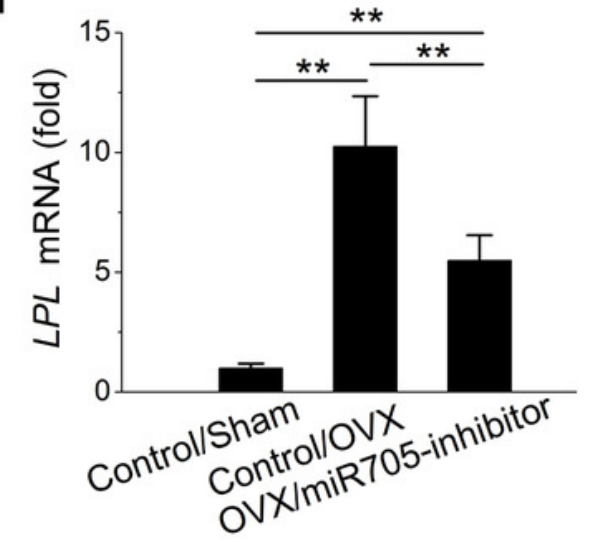




\section{Figure 8}

Knockdown of miR-705 increases the target genes expression in MSCs from osteoporotic mandibles.

Sham MSCs were transfected with the reagent as a negative control. OVX MSCs were transfected with the reagent and miR-705 inhibitor. miR-705 expression in three groups was measured by qRT-PCR at $48 \mathrm{~h}$ post-transfection (a). HOXA10 and FoxO1 expression was measured by western blot (b). Values are described as the mean \pm SD from three independent experiments. $* P<0.01, * * P<0.001$.

a

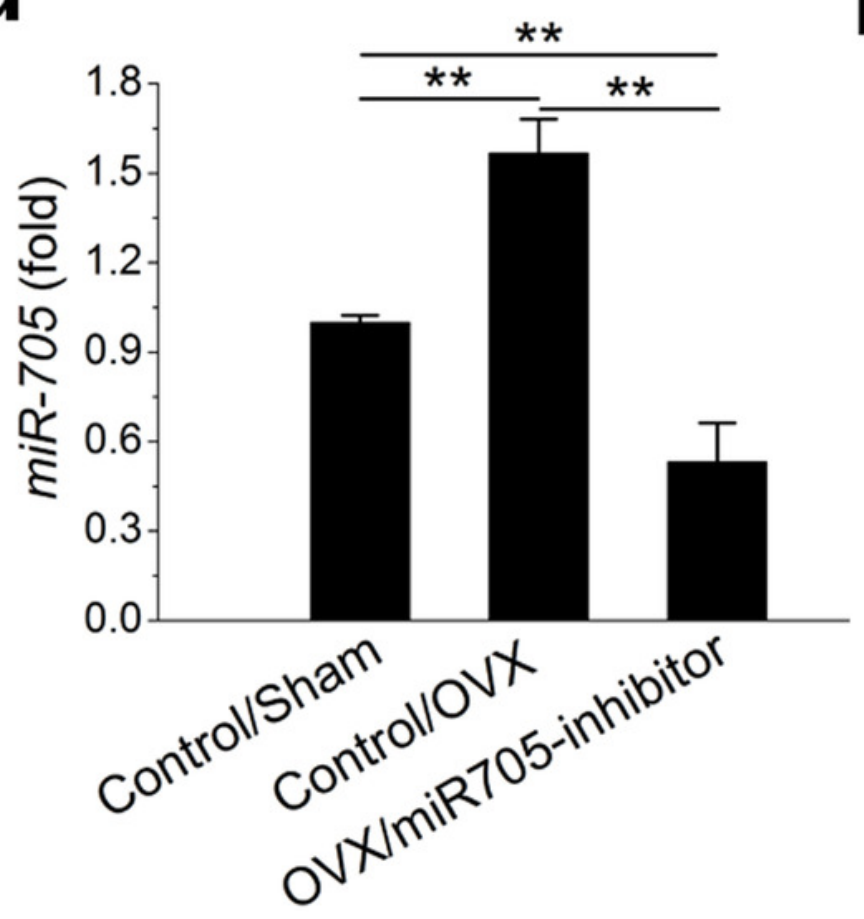

HOXA10

FoxO1

$\beta$-actin
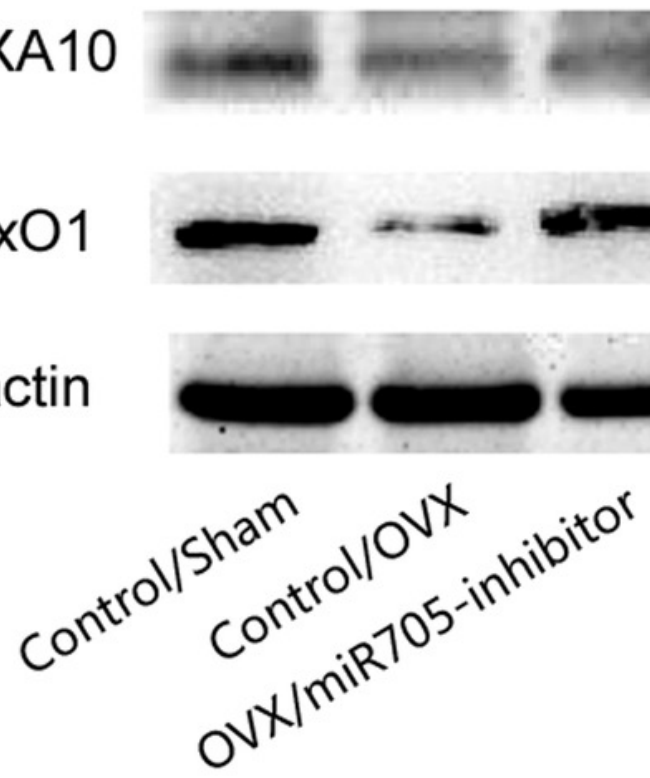